\title{
Peripheral inflammation/immune indicators of chemosensitivity and prognosis in breast cancer patients treated with neoadjuvant chemotherapy
}

This article was published in the following Dove Press journal:

OncoTargets and Therapy

Yi Qian',*
Jing Tao',2,*
Xiuqing Li'
Hua Chen'
Qi Lu'
Junzhe Yang'
Hong Pan'
Cong Wang ${ }^{4}$
Wenbin Zhou'
Xiaoan Liu'
'Department of Breast Surgery, The
First Affiliated Hospital with Nanjing
Medical University, Nanjing, China;
${ }^{2}$ Department of General Surgery,
Nanjing Pukou Hospital, Nanjing,
China; ${ }^{3}$ Department of Pathology,
Jiangsu Province Hospital of
Traditional Chinese Medicine, Nanjing,
China; ${ }^{4}$ Department of Pathology, The
First Affiliated Hospital with Nanjing
Medical University, Nanjing, China
*These authors contributed equally
to this work

to this work

\begin{abstract}
Introduction: Neoadjuvant chemotherapy (NAC) has become a standard treatment for locally advanced breast cancer. The present study was designed to investigate the predictive value of different peripheral inflammation/immune biomarker responses to NAC and prognosis in breast cancer patients.
\end{abstract}

Materials and methods: A total of 180 breast cancer patients treated with NAC in the First Affiliated Hospital with Nanjing Medical University between January 2008 and March 2015 were enrolled in the study. The associations between inflammation/immune indicators and pathological complete response (pCR) were determined, and the prognostic value of inflammation/immune indicators was also evaluated.

Results: In the univariate analysis, patients with a high pretreatment peripheral lymphocyte count $\left(>2.06 \times 10^{9} / \mathrm{L}\right)$ showed a higher $\mathrm{pCR}$ rate than those with a low lymphocyte count $(23.9 \%$ vs $10.4 \%, P=0.023)$. The $\mathrm{pCR}$ rate of patients with a neutrophil: lymphocyte ratio $\leq 2.15$ was significantly higher than that of patients with a high neutrophil: lymphocyte ratio ( $20 \% \mathrm{vs} 7.8 \%$; $P=0.03)$. However, multivariate analysis revealed that only the high lymphocyte count was predictive for pCR (odds ratio: $4.375,95 \% \mathrm{CI}$ : 1.429-13.392, $P=0.010$ ). In the survival analysis, patients with a higher neutrophil count $\left(>2.65 \times 10^{9} / \mathrm{L}\right)$ were confirmed to have a shorter diseasefree survival (hazard ratio: 4.322, 95\% CI: 1.028-18.174, $P=0.046$ ), and the high neutrophil count was significantly associated with lymphovascular invasion $(P=0.037)$.

Conclusion: We demonstrated that a high level of baseline peripheral lymphocyte count can be a predictor for high efficacy of NAC for breast cancer patients, and low baseline peripheral neutrophil count may contribute to the favorable disease-free survival.

Keywords: breast cancer, lymphocytes, neoadjuvant chemotherapy, neutrophils, pathologic complete response

\section{Introduction}

Breast cancer is one of the most common malignancies among women nowadays. ${ }^{1-3}$ For patients with locally advanced breast cancer, neoadjuvant chemotherapy (NAC) has become a standard treatment, which can reduce the tumor burden and allow a rapid assessment of drug susceptibility preoperatively. 3,4 Patients with pathological complete response ( $\mathrm{pCR}$ ) to NAC are considered to have a longer disease-free survival (DFS) than those without pCR. ${ }^{5}$ However, patients with hormone receptor-positive and human epidermal growth factor receptor 2 (Her2) negative breast cancer have shown a lower pCR rate than those with triple-negative and Her2-enriched cancer, but with a better clinical outcome. ${ }^{6,7}$ Thus, effective indictors are still required to predict the response to drugs for appropriate chemotherapy regimens. Moreover, prognostic
Correspondence: Wenbin Zhou;

Xiaoan Liu

Department of Breast Surgery,

The First Affiliated Hospital, Nanjing

Medical University, 300 Guangzhou Road,

210029 Nanjing, China

Tel +862583718836 ext 862 I

Fax +862583718836

Email zhouwenbin@njmu.edu.cn;

liuxiaoan@I26.com
OncoTargets and Therapy 2018: | | |423-|432

1423

Dovepress If in

http://dx.doi.org/10.214710TTS1 48496 (c) (7) (5) 2018 Qian et al. This work is published and licensed by Dove Medical Press Limited. The full terms of this license are available at https://www.dovepress.com/terms.php (c) ${ }_{\mathrm{BY}} \mathrm{NC}$ and incorporate the Creative Commons Attribution - Non Commercial (unported, v3.0) License (http://(creativecommons.org/licenses/by-n/ 3.00 ). By accessing the work you hereby accept the Terms. Non-commercial uses of the work are permitted without any further permission from Dove Medical Press Limited, provided the work is properly attributed. For permission for commercial use of this work, please see paragraphs 4.2 and 5 of our Terms (https://www.dovepress.com/terms.php). 
markers related to relapse are crucial for further treatment, especially in patients with residual disease after NAC.

Advancement in the development of cancer biology and rapid progress of experimental technology has led to a growing number of factors being proposed in the past decade to predict the drug efficiency and clinical outcome of breast cancer patients. Besides the general clinicopathologic features ${ }^{8}$ recent advances in breast cancer-related genes (bcl-2, ras, p53, etc.) provide significant information on curative effect and prognosis. ${ }^{9-13}$ Due to the complexity of the technological process and the high cost, it is difficult to use genetic test widely in clinical practice. With the introduction of tumor microenvironment, ${ }^{14,15}$ there has been a worldwide upsurge of research interest in tumor-infiltrating immune cells in recent years. ${ }^{16,17}$ The level of tumor-infiltrating immune cells, especially the tumor-infiltrating lymphocytes (TILs), has been confirmed to be an important indicator of chemosensitivity and survival due to its role in cell-mediated immunologic destruction of tumor cells. ${ }^{18}$ However, as TILs mainly work in situ, they can hardly represent the immune response of the whole body against breast cancer, which is considered as a systemic disease. Therefore, new systemic markers should be found out to predict the response and prognosis easily and accurately.

Recently, inflammation and immune-based indicators in the peripheral blood, closely linked with immune/inflammatory response against tumors, have been developed as predictive factors for chemosensitivity and survival for several tumors. ${ }^{19-23}$ It has been reported that an elevated neutrophil count is associated with a poor prognosis in non-small-cell lung cancer, ${ }^{24}$ and a high peripheral lymphocyte count is related to a favorable outcome in advanced sarcomas and lymphomas. ${ }^{19}$ Furthermore, the neutrophil: lymphocyte ratio (NLR) has been recognized as an important predictor of prognosis in colorectal cancer, urothelial cancer, and gastric cancer, ${ }^{21-23}$ and NLR is associated with the response to NAC in muscle-invasive bladder cancer. ${ }^{25}$ To the best of our knowledge, the roles of these biomarkers remain unclear in breast cancer patients. ${ }^{26-29}$ The objective of our present research was to evaluate the predictive value of different immune/inflammatory markers in drug efficiency and prognosis in breast cancer patients.

\section{Materials and methods}

\section{Patients and treatments}

In this retrospective study, patients were enrolled from the First Affiliated Hospital with Nanjing Medical University between January 2008 and March 2015, with the inclusion criteria as listed: 1) diagnosed with invasive breast cancer by core biopsy, 2) diagnosed as stage II-III, and 3) treated with chemotherapy followed by surgery. All the clinicopathological data, such as the patients' age, laboratory findings, and pathologic results, were obtained from the hospital's database with the approval of the ethics committee of the First Affiliated Hospital with Nanjing Medical University.

Laboratory variables were retrieved from blood tests taken within 1 week before NAC through the hematology analyzer Sysmex XS-800i (Sysmex Corporation, Kobe, Japan), to achieve information on immune/inflammatory indicators, including neutrophil counts, lymphocyte counts, and the ratio between them. No patients had infection, such as pneumonia.

All patients received taxane- and/or anthracyclinebased chemotherapy regimens in neoadjuvant settings: a total of 4 courses of epirubicin+cyclophosphamide (90 mg/m² epirubicin and $600 \mathrm{mg} / \mathrm{m}^{2}$ cyclophosphamide), followed by 4 courses of docetaxel (100 $\mathrm{mg} / \mathrm{m}^{2}$ docetaxel) every 2 or 3 weeks; 6 courses of docetaxel+epirubicin+ cyclophosphamide $\left(75 \mathrm{mg} / \mathrm{m}^{2}\right.$ docetaxel, $90 \mathrm{mg} / \mathrm{m}^{2}$ epirubicin, and $500 \mathrm{mg} / \mathrm{m}^{2}$ cyclophosphamide) every 3 weeks; 6 courses of docetaxel+cyclophosphamide $\left(100 \mathrm{mg} / \mathrm{m}^{2}\right.$ docetaxel and $600 \mathrm{mg} / \mathrm{m}^{2}$ cyclophosphamide) every 3 weeks; 6 courses of fluorouracil+epirubicin+cyclophosphamide $\left(500 \mathrm{mg} / \mathrm{m}^{2}\right.$ fluorouracil, $100 \mathrm{mg} / \mathrm{m}^{2}$ epirubicin, and $500 \mathrm{mg} / \mathrm{m}^{2}$ cyclophosphamide) every 3 weeks; and 6 courses of docetaxel+carboplatin+ trastuzumab $\left(75 \mathrm{mg} / \mathrm{m}^{2}\right.$ docetaxel, carboplatin: area under the curve $=5$, trastuzumab: $8 \mathrm{mg} / \mathrm{kg}$ followed by $6 \mathrm{mg} / \mathrm{kg}$ ) every 3 weeks. If Her 2 was amplified, trastuzumab was then recommended to be added. However, due to the high cost, about $40 \%$ of patients with Her2-positive cancer were treated with trastuzumab. Surgery was performed within 3 weeks after the last course of chemotherapy, including modified mastectomy, radical mastectomy, and breast-conserving surgery, followed by radiotherapy and endocrine therapy if needed. NAC, surgery, and the subsequent radiotherapy and endocrine therapy were recommended to the patients according to the National Comprehensive Cancer Network $(\mathrm{NCCN})$ guidelines.

\section{Pathology}

Patients were diagnosed by core biopsy before treatment. Imaging technologies, including CT, ultrasonography (US), MRI, and bone scintigraphy, were applied for evaluation. Tumor stage was assessed according to the TNM Classification of Malignant Tumors published by the American Joint Committee on Cancer. Pathologic results, such as estrogen receptor (ER), progesterone receptor (PR), Her2, and Ki67, 
were assessed by immunohistochemistry. ER and PR were considered positive when at least $1 \%$ of tumor cell nuclei were immunoreactive based on the American Society of Clinical Oncology (ASCO)/College of American Pathologists (CAP) guideline recommendations for immunohistochemical testing of ER and PR. Her2 was defined as positive if the score was $\geq 3$ by immunohistochemistry or $\geq 2$ with further analysis by fluorescence in situ hybridization according to the ASCO/ CAP HER2 testing guideline. Considering that cutoff of Ki67 varies between different pathological experiment centers, we defined it at a high level if the Ki67 proliferation index was $>20 \%$ according to our institution. Molecular subtype was defined based on the NCCN and St Gallen guidelines. pCR was defined as no evidence of invasive cancer residue in the surgical specimen and all axillary lymph nodes being free of tumor, according to the Response Evaluation Criteria in Solid Tumors criteria of resected primary tumors.

\section{Follow-up}

Patients were followed up every 3 months during the first 2 postoperative years and every 6 months until 5 years after surgery, and then every 12 months. Patients were monitored by imaging studies, such as blood tests, US, CT, and bone scintigraphy. Once local regional recurrence or distant metastasis was suspected, further examination was recommended. The follow-up time was calculated from the date of diagnosis to the date of relapse or last follow-up.

\section{Statistical analysis}

Receiver operating characteristic (ROC) curve analysis was used to determine the cutoff of neutrophil and lymphocyte counts, and the cutoff of NLR was determined by its median value. The association between clinicopathological factors and pretreatment immune/inflammatory indicators, and the predictive value of different variables for $\mathrm{PCR}$ in the univariate analysis were assessed by using the chi-square test. The Fisher's exact test was used if the expected frequency was $<5$. Multivariate binary logistic regression was performed to identify the factors that were independently correlated with $\mathrm{pCR}$ rate. For survival analysis, we applied the Kaplan-Meier method as univariate analysis and Cox proportional hazard model as multivariate analysis. The result was considered significant when the $P$-value was $<0.05$. Statistical analyses were performed using the SPSS software (version 21.0; SPSS Inc, Chicago, IL, USA).

\section{Ethical approval}

All procedures performed in studies involving human participants received approval of the ethics committee of the
First Affiliated Hospital with Nanjing Medical University and were in accordance with the 1964 Helsinki Declaration and its later amendments or comparable ethical standards. Written informed consent was obtained from all individual participants included in the study.

\section{Results}

\section{Patient enrollment}

A total of 248 breast cancer patients were screened in our study. Patients without operation after NAC and with bilateral breast cancer or second primary cancer were excluded. Of the remaining breast cancer patients who had completed the primary treatment, patients with systemic inflammatory or chronic diseases preoperatively, such as diabetes, heart failure, liver cirrhosis, and systemic lupus erythematosus, or patients with missing information about pathologic or laboratory results were excluded. Therefore, 180 subjects met the inclusion criteria for pCR analysis. And then, patients with incomplete adjuvant radiotherapy or endocrine therapy after surgery were filtered out, and patients who were lost during follow-up were excluded. Finally, 131 female breast cancer patients were included in the survival analysis. The flowchart of patients' screening is shown in Figure 1.

The baseline characteristics of the including patients are shown in Table 1. The median age at diagnosis of the subjects was 46 years old (range, 23-71 years). Of these 180 patients, $86.1 \%$ were diagnosed with invasive ductal carcinoma; $58.7 \%$ were diagnosed with hormone receptor-positive cancer at initial diagnosis by core needle biopsy; $21.1 \%$ were Her2 positive; and $63.6 \%$ were diagnosed with high Ki67 expression (positive Ki67 staining $>20 \%$ ) cancer. Surgery was performed on all patients after NAC, and about $90 \%$ of them received modified radical mastectomy. Axillary lymph node involvement and lymph vessel invasion were found in $39.5 \%$ and $22.2 \%$ of these cases, respectively.

\section{Associations of immune/inflammatory indicators with clinicopathological parameters}

The mean absolute pretreatment neutrophil count was $3.95 \times 10^{9} / \mathrm{L}$ (range, $0.73-12.89 \times 10^{9} / \mathrm{L}$ ). The optimal cutoff value of neutrophil count was $2.65 \times 10^{9} / \mathrm{L}$, determined as the maximum (sensitivity + specificity) point according to ROC curves. Among the 180 patients, 34 patients had a neutrophil count of $\leq 2.65 \times 10^{9} / \mathrm{L}$, and 146 patients had a neutrophil count of $>2.65 \times 10^{9} / \mathrm{L}$. The high neutrophil count was significantly associated with lymph vessel invasion $(P=0.037)$, while no significant associations were detected between the neutrophil count and other clinicopathological parameters. 


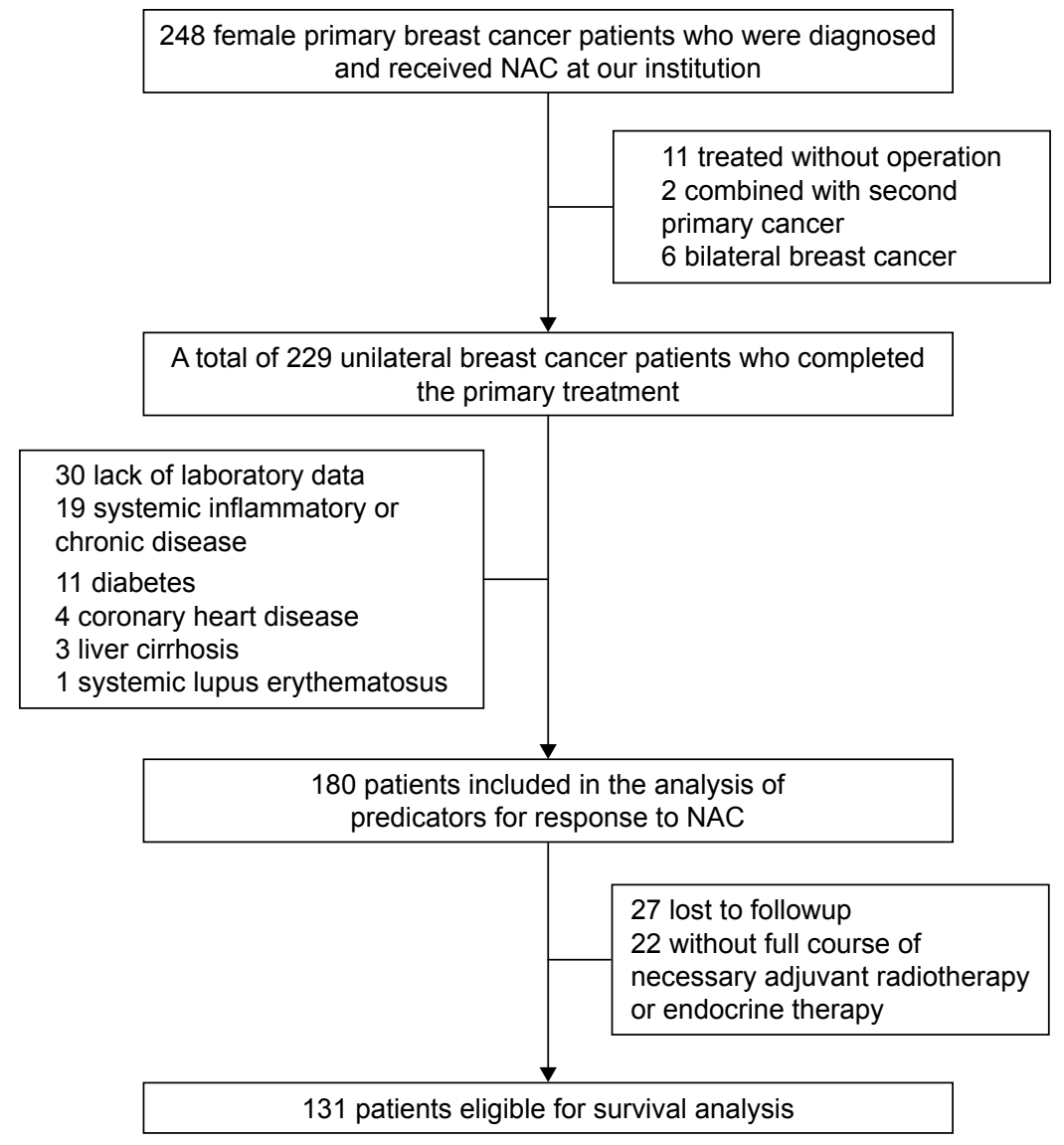

Figure I Flowchart of screening patients eligible in the study.

Abbreviation: NAC, neoadjuvant chemotherapy.

The mean absolute pretreatment lymphocyte count was $1.75 \times 10^{9} / \mathrm{L}$ (range, $0.80-3.90 \times 10^{9} / \mathrm{L}$ ). The cutoff $\left(2.06 \times 10^{9} / \mathrm{L}\right)$ to define the high lymphocyte count group $\left(>2.06 \times 10^{9} / \mathrm{L}\right)$, and the low lymphocyte count group was determined by ROC curve analysis. There were 134 patients with a lymphocyte count of $\leq 2.06 \times 10^{9} / \mathrm{L}$, and 46 patients whose lymphocyte count was $<2.06 \times 10^{9} / \mathrm{L}$. No significant associations were observed between the lymphocyte count and clinicopathological parameters.

The mean NLR was 2.44 , and the median value (2.15) was used as the cutoff. No significant association was found between NLR and clinicopathological parameters.

\section{Relationships between immune/ inflammatory indicators and pathologic response to chemotherapy}

The correlations between clinicopathological factors and pathologic response are shown in Table 2. Of the 180 patients, $25(13.9 \%)$ achieved pCR. In the univariate analysis, ER status $(P=0.003)$, molecular subtype $(P=0.018)$, and Ki67 proliferation index $(P=0.043)$ were significantly associated with the response to NAC. Moreover, high pretreatment lymphocyte count was detected to be a significant predictor $(P=0.023)$ of $\mathrm{pCR}$ in the present study. There were 14 complete responders among the 134 patients with a baseline lymphocyte count of $\leq 2.06 \times 10^{9} / \mathrm{L}$ and 11 responders among the 46 patients with a baseline lymphocyte count of $>2.06 \times 10^{9} / \mathrm{L}$. Moreover, the $\mathrm{pCR}$ rate of the patients with an NLR of $\leq 2.15$ was significantly higher than that of the patients with a high NLR of $>2.15(20 \%$ vs $7.8 \% ; P=0.03)$.

Then, lymphocyte count, NLR, ER status, molecular subtype, and Ki67 proliferation index were incorporated into the multivariate binary logistic regression analysis. It was confirmed that lymphocyte count remained an independent predictor of response to chemotherapy with an odds ratio (OR) of $4.375(95 \%$ CI: 1.429-13.392, $P=0.010$ ), along with ER status (OR: 0.203, 95\% CI: 0.067-0.614, $P=0.003$ ) and Ki67 proliferation index (OR: 6.290, 95\% CI: 1.495-26.461, $P=0.012$ ). However, the NLR was not a predictor of $\mathrm{pCR}$ in the multivariate analysis.

\section{Predictive value of immune/inflammatory indicators in view of survival}

With a mean follow-up of 22.7 months (range, 3-85 months), $37(28.2 \%)$ patients showed local-regional recurrence or 
Table I Baseline characteristics of 180 patients with breast cancer

\begin{tabular}{|c|c|}
\hline Characteristics & $\mathbf{N}(\%)$ \\
\hline \multicolumn{2}{|l|}{ Age (years) } \\
\hline$\leq 40$ & $46(25.6)$ \\
\hline$>40$ & I $34(74.4)$ \\
\hline \multicolumn{2}{|l|}{ Clinical T stage } \\
\hline TI & $15(8.3)$ \\
\hline $\mathrm{T} 2$ & $96(53.3)$ \\
\hline T3-T4 & $55(30.6)$ \\
\hline NA & $14(7.8)$ \\
\hline \multicolumn{2}{|l|}{ Clinical $N$ stage } \\
\hline No & $43(23.9)$ \\
\hline $\mathrm{NI}-\mathrm{N} 3$ & I $37(76.1)$ \\
\hline \multicolumn{2}{|l|}{ Histology } \\
\hline Ductal & I55 (86. I) \\
\hline Lobular & $3(1.67)$ \\
\hline Others & $22(12.2)$ \\
\hline \multicolumn{2}{|l|}{ Estrogen receptor status } \\
\hline Positive & $84(46.7)$ \\
\hline Negative & $59(32.8)$ \\
\hline NA & $37(20.5)$ \\
\hline \multicolumn{2}{|l|}{ Her2 status } \\
\hline Negative & $112(62.2)$ \\
\hline Positive & $30(16.7)$ \\
\hline NA & $38(2 \mathrm{I} . \mathrm{I})$ \\
\hline \multicolumn{2}{|l|}{ Molecular subtype } \\
\hline Luminal A & $24(13.3)$ \\
\hline Luminal B & $60(33.3)$ \\
\hline Her2 & $18(10.0)$ \\
\hline Triple negative & $40(22.2)$ \\
\hline NA & $38(21.2)$ \\
\hline \multicolumn{2}{|l|}{ Ki67 proliferation index } \\
\hline$\leq 20$ & $51(28.3)$ \\
\hline$>20$ & $89(49.4)$ \\
\hline NA & $40(22.3)$ \\
\hline \multicolumn{2}{|l|}{ NAC regimen } \\
\hline Anthra based & $42(23.3)$ \\
\hline Tax based & $12(6.7)$ \\
\hline Anthra + Tax based & $126(70.0)$ \\
\hline \multicolumn{2}{|l|}{ Surgery } \\
\hline Modified mastectomy & I64 (9I.I) \\
\hline Radical mastectomy & $13(7.2)$ \\
\hline Breast-conserving surgery & $3(1.7)$ \\
\hline \multicolumn{2}{|c|}{ Pathological $\mathrm{N}$ stage after surgery } \\
\hline No & $107(59.4)$ \\
\hline $\mathrm{NI}$ & $5(2.8)$ \\
\hline N2 & $7(3.9)$ \\
\hline N3 & $58(32.2)$ \\
\hline NA & $3(1.7)$ \\
\hline \multicolumn{2}{|l|}{ Lymph vessel invasion } \\
\hline Yes & $40(22.2)$ \\
\hline No & $140(77.8)$ \\
\hline
\end{tabular}

Abbreviations: Anthra, anthracyclines; NA, not available; NAC, neoadjuvant chemotherapy; Tax, taxanes.

distant metastasis. Pretreatment neutrophil count, vascular invasion, and age were all associated with DFS in the univariate analysis (Table 3 ). Significantly longer (log-rank $P=0.029)$ DFS was observed in the low neutrophil count group (pretreatment neutrophil count $\leq 2.65 \times 10^{9} / \mathrm{L}$ ) compared with the high neutrophil count group (Figure 2), while no significant association was found between DFS and other immune/inflammatory indicators.

Variables, including age, lymph vessel invasion, and neutrophil count, with a $P$-value $<0.05$ in the univariate analysis, were further involved in the multivariate analysis. The Cox proportional multivariate hazard model was applied. The higher neutrophil count along with younger age at diagnosis was independently correlated with poor prognosis, with a hazard ratio of 4.322 (95\% CI: $1.028-18.174, P=0.046)$ and 2.165 (95\% CI: $1.127-4.149, P=0.02$ ), respectively (Table 3).

Because of the small sample size and the relatively short follow-up, no significant associations were observed between clinicopathological factors and overall survival (data not shown).

\section{Discussion}

The present study focused on the predictive value of different immune/inflammatory indicators in chemosensitivity and survival in breast cancer patients treated with NAC. It has been shown that a high pretreatment lymphocyte count and a relatively low NLR rate were significantly associated with pCR in the univariate analysis, while only the pretreatment lymphocyte count acted as an independent predictor of $\mathrm{pCR}$ in the multivariate model. In addition, an elevated neutrophil count, which was associated with lymph vessel invasion after NAC, was confirmed to be correlated with poor DFS independently.

Consistent with previous studies, ${ }^{30}$ subgroups with higher proliferation, Her2-positive (non-luminal), and triple-negative tumors were confirmed more likely to achieve pCR in this study. Of hormone receptor-positive cancer, the $\mathrm{pCR}$ rate was relatively low in the present analysis. Considering that only a small proportion of patients with Her2-positive status were treated with trastuzumab in the neoadjuvant setting, the pCR rate of Her2-positive patients in our study was not so high as in studies with trastuzumab-based NAC. ${ }^{31}$

Interestingly, a significant association was demonstrated between a high absolute baseline peripheral lymphocyte count and pCR in our study, independent of the general pathological characteristics of tumors. Until now, only few research have raised concerns about the impact of pretreatment peripheral immune/inflammatory indicators on pCR. ${ }^{26,28,29}$ Pretreatment lymphocytopenia has been recognized as a negative marker on the efficacy of preoperative chemotherapy in muscle-invasive and advanced bladder cancer. ${ }^{32}$ While in breast cancer patients, as far as we know, the present study was the first to propose the predictive impact of the 
Table 2 Predictive factors for pathological complete response in univariate and multivariate analyses

\begin{tabular}{|c|c|c|c|c|c|c|}
\hline Variables & Total, $\mathbf{N}$ & $\begin{array}{l}\text { Non-pCR } \\
\text { group (N) }\end{array}$ & $\begin{array}{l}\text { PCR group } \\
(\mathrm{N}, \%)\end{array}$ & $P$-value ${ }^{a}$ & $\begin{array}{l}\text { Multivariate OR } \\
(95 \% \mathrm{CI})^{\mathrm{b}}\end{array}$ & $P$-value ${ }^{b}$ \\
\hline Age (years) & & & & 0.028 & & 0.055 \\
\hline$\leq 40$ & 46 & 44 & $2(4.30)$ & & & \\
\hline$>40$ & 134 & 11 & $23(17.2)$ & & & \\
\hline Clinical T stage & & & & 0.803 & & \\
\hline TI & 15 & 12 & $3(20)$ & & & \\
\hline $\mathrm{T} 2$ & 96 & 83 & $13(13.50)$ & & & \\
\hline T3-T4 & 55 & 47 & $8(14.5)$ & & & \\
\hline NA & 14 & 13 & I & & & \\
\hline Clinical $N$ stage & & & & 0.603 & & \\
\hline No & 43 & 36 & $7(28.0)$ & & & \\
\hline $\mathrm{NI}-\mathrm{N} 3$ & 137 & 119 & $18(72.0)$ & & & \\
\hline Estrogen receptor & & & & 0.003 & & 0.005 \\
\hline Positive & 84 & 78 & $6(7.10)$ & & $0.203(0.067-0.614)$ & \\
\hline Negative & 59 & 44 & $15(25.40)$ & & & \\
\hline NA & 37 & 31 & 6 & & & \\
\hline Her2 status & & & & 0.38 & & \\
\hline Negative & 112 & 97 & $15(13.40)$ & & & \\
\hline Positive & 30 & 24 & $6(20.00)$ & & & \\
\hline NA & 38 & 32 & 6 & & & \\
\hline Molecular subtype & & & & 0.018 & & 0.606 \\
\hline Luminal A & 24 & 23 & I (4.20) & & & \\
\hline Luminal B & 60 & 55 & $5(8.30)$ & & & \\
\hline Her2 & 18 & 13 & $5(27.80)$ & & & \\
\hline Triple-negative & 40 & 30 & $10(25.00)$ & & & \\
\hline NA & 38 & 32 & 6 & & & \\
\hline Ki67 proliferation index & & & & 0.043 & & 0.012 \\
\hline$\leq 20$ & 51 & 48 & $3(5.90)$ & & $6.290(\mathrm{I} .495-26.46 \mathrm{I})$ & \\
\hline$>20$ & 89 & 72 & $17(19.10)$ & & & \\
\hline NA & 40 & 33 & 7 & & & \\
\hline NAC regimens & & & & 0.619 & & \\
\hline Anthra based & 42 & 38 & $4(9.50)$ & & & \\
\hline Tax based & 12 & 10 & $2(16.7)$ & & & \\
\hline Anthra + Tax based & 126 & 107 & $19(15.1)$ & & & \\
\hline Lymphocyte counts & & & & 0.023 & & 0.010 \\
\hline$\leq 2.06 \times 10^{9} / \mathrm{L}$ & 134 & 120 & $14(10.4)$ & & $4.375(1.429-13.392)$ & \\
\hline$>2.06 \times 10^{9} / \mathrm{L}$ & 46 & 35 & II (23.9) & & & \\
\hline Neutrophil counts & & & & 0.686 & & \\
\hline$\leq 2.65 \times 10^{9} / \mathrm{L}$ & 34 & 30 & $4(11.80)$ & & & \\
\hline$>2.65 \times 10^{9} / \mathrm{L}$ & 146 & 125 & $21(14.4)$ & & & \\
\hline NLR & & & & 0.03 & & 0.254 \\
\hline$\leq 2.15$ & 90 & 72 & $18(20)$ & & & \\
\hline$>2.15$ & 90 & 83 & $7(7.8)$ & & & \\
\hline
\end{tabular}

Notes: ${ }^{a}$ Calculated by chi-square test as univariate analyses. ${ }^{b}$ Calculated by multivariate binary logistic regression analysis.

Abbreviations: Anthra, anthracyclines; NA, not available; NAC, neoadjuvant chemotherapy; NLR, neutrophil-to-lymphocyte ratio; OR, odds ratio; pCR, pathological complete response; Tax, taxanes.

peripheral lymphocyte count on pCR. Previous studies have reported that the level of TILs is a strong predictor of the efficacy of NAC, ${ }^{16}$ particularly in breast cancer patients with triple-negative or Her2-positive (non-luminal) subtypes. ${ }^{33,34}$ However, the inability to represent the whole-body immune response limits the application of TILs in predicting the systematic therapeutic effects. Studies on the corresponding peripheral immune/inflammatory indicators seem to be fascinating. Lymphocytes, which have been demonstrated as a heterogeneous population with the cytotoxic $\mathrm{T}$ cells, with CD8 expressed on the cell surface being the predominant $\mathrm{T}$ lymphocyte present in most cases, form a major effector component of the adaptive immune system. The CD8+ $T$ cells can limit tumor growth by recognizing tumor-specific antigens expressed by the cancer cells and stimulating the apoptosis of cancer cells by classical cytotoxic immune 
Table 3 Survival analysis for disease-specific survival in I3I patients

\begin{tabular}{|c|c|c|c|}
\hline Characteristics & $\begin{array}{l}\text { Univariate } \\
P \text {-value }{ }^{a}\end{array}$ & $\begin{array}{l}\text { Multivariate HR } \\
(95 \% \mathrm{Cl})^{\mathrm{b}}\end{array}$ & $P$-value \\
\hline Age ( $\leq 40$ years vs $>40$ years) & 0.029 & $2.165(1.127-4.149)$ & 0.02 \\
\hline Clinical T stage & 0.109 & & \\
\hline Clinical N stage (N0 vs NI-N3) & 0.533 & & \\
\hline Estrogen receptor (positive vs negative) & 0.193 & & \\
\hline Her2 status (positive vs negative) & 0.152 & & \\
\hline Molecular subtype & 0.182 & & \\
\hline Ki67 proliferation index ( $\leq 20$ vs $>20)$ & 0.091 & & \\
\hline Nuclear grade & 0.249 & & \\
\hline Lymph node status & 0.096 & & \\
\hline Lymph vessel invasion (yes vs no) & 0.039 & $1.948(0.989-3.838)$ & 0.054 \\
\hline Adjuvant radiotherapy (no vs yes) & 0.232 & & \\
\hline Adjuvant chemotherapy (Anthra based vs Tax based vs Anthra + Tax based) & 0.718 & & \\
\hline Lymphocyte counts $\left(\leq 2.06 \times 10^{9} / \mathrm{L}\right.$ vs $\left.>2.06 \times 10^{9} / \mathrm{L}\right)$ & 0.880 & & \\
\hline Neutrophil counts $\left(>2.65 \times 10^{9} / \mathrm{L}\right.$ vs $\left.\leq 2.65 \times 10^{9} / \mathrm{L}\right)$ & 0.029 & $4.322(1.028-18.174)$ & 0.046 \\
\hline $\operatorname{NLR}(\leq 2.15$ vs $>2.15)$ & 0.535 & & \\
\hline
\end{tabular}

Note: a Derived using the Kaplan-Meier method as univariate analysis, berived using the Cox regression adjusted for age at diagnosis and neutrophil counts.

Abbreviations: Anthra, anthracyclines; HR, hazard ratio; NLR, neutrophil-to-lymphocyte ratio; Tax, taxanes.

effector mechanisms both in the tumor and in the peripheral blood. ${ }^{35}$ By contrast, tumors can also promote immunosuppressive regulatory $\mathrm{T}$ cells accumulating in the tumor or in the peripheral blood, in which the nuclear transcription factor known as FOXP3 is the most specific cell marker identified. However, the role of FOXP3 in tumor has remained controversial. In addition, recent researches proposed that the immune cells in the peripheral blood can also contribute to establishing and maintaining a status of tumor dormancy, in which tumor cells would be present, but without apparent progression. ${ }^{36}$ Although the cytotoxic immune cells around the tumor cells, before systematic treatment, were found mostly inactive in locally advanced cancer, it is assumed that chemotherapy can activate the adaptive immune system and sensitize tumor cells to T-cell-mediated cytotoxicity by improving the efficacy of perforin/granzyme-mediated or Fas ligand-mediated killing. ${ }^{18,37} \mathrm{~A}$ high pretreatment peripheral lymphocyte count is therefore a substitute for a high anticancer potential of the systemic immunity, which might predict a better response to chemotherapy.

NLR is one of the peripheral immune/inflammatory indicators mostly reported in breast cancer. Several studies have suggested that a lower baseline NLR is correlated with a higher likelihood of pCR. ${ }^{26,27}$ However, no relationship has been observed in other studies. ${ }^{28,29}$ The present study found that the pretreatment NLR was a predictive factor of $\mathrm{pCR}$ in the univariate analysis, but not in the multivariate analysis. Considering the irrelevance of the peripheral neutrophil count to $\mathrm{pCR}$, the predictive value of a low NLR may be mainly driven by the high lymphocyte count actually.
In several studies, $\mathrm{pCR}$ has been considered as a predictor for favorable outcome. ${ }^{5}$ However, breast cancer patients in hormone receptor-positive and Her2-negative subgroups, with a lower $\mathrm{pCR}$ rate than triple-negative and Her2-positive ones, show a better clinical outcome instead. ${ }^{6,7}$ Containing all molecular subtypes may attenuate the prognostic value of pCR. ${ }^{30}$ With a relatively small sample size and mixed subtypes included in our analysis, the achievement of pCR showed no significant benefit for DFS.

The baseline peripheral neutrophil count was demonstrated as an independent prognostic factor in our study. It showed that a high neutrophil count had an apparent correlation with a poor prognosis, which was in accordance with studies on other cancers. ${ }^{24,38}$ Previous studies have shown that neutrophils occupy a significant portion of the inflammatory response, which is usually considered as a pro-tumor response. The underlying mechanisms are as follows: First, neutrophils-derived reactive oxygen species have been implicated with genotoxic effects to promote tumor establishment. Additionally, the chemokines and/or cytokines produced by neutrophils, such as interleukin 6-like cytokine oncostatin $\mathrm{M}$, may activate series inflammatory factors, including nuclear factor- $\mathrm{K} \mathrm{B}$, pro-angiogenic factors (vascular endothelial growth factor), etc., which are detected with a negative impact on tumor immune surveillance and lead to tumor progression. And the overexpression of human neutrophil-derived defensins has been identified in several tumor types as having association with tumor cell proliferation and invasiveness. ${ }^{39}$ Moreover, the tumor-associated neutrophils have been recognized to play an important role in 

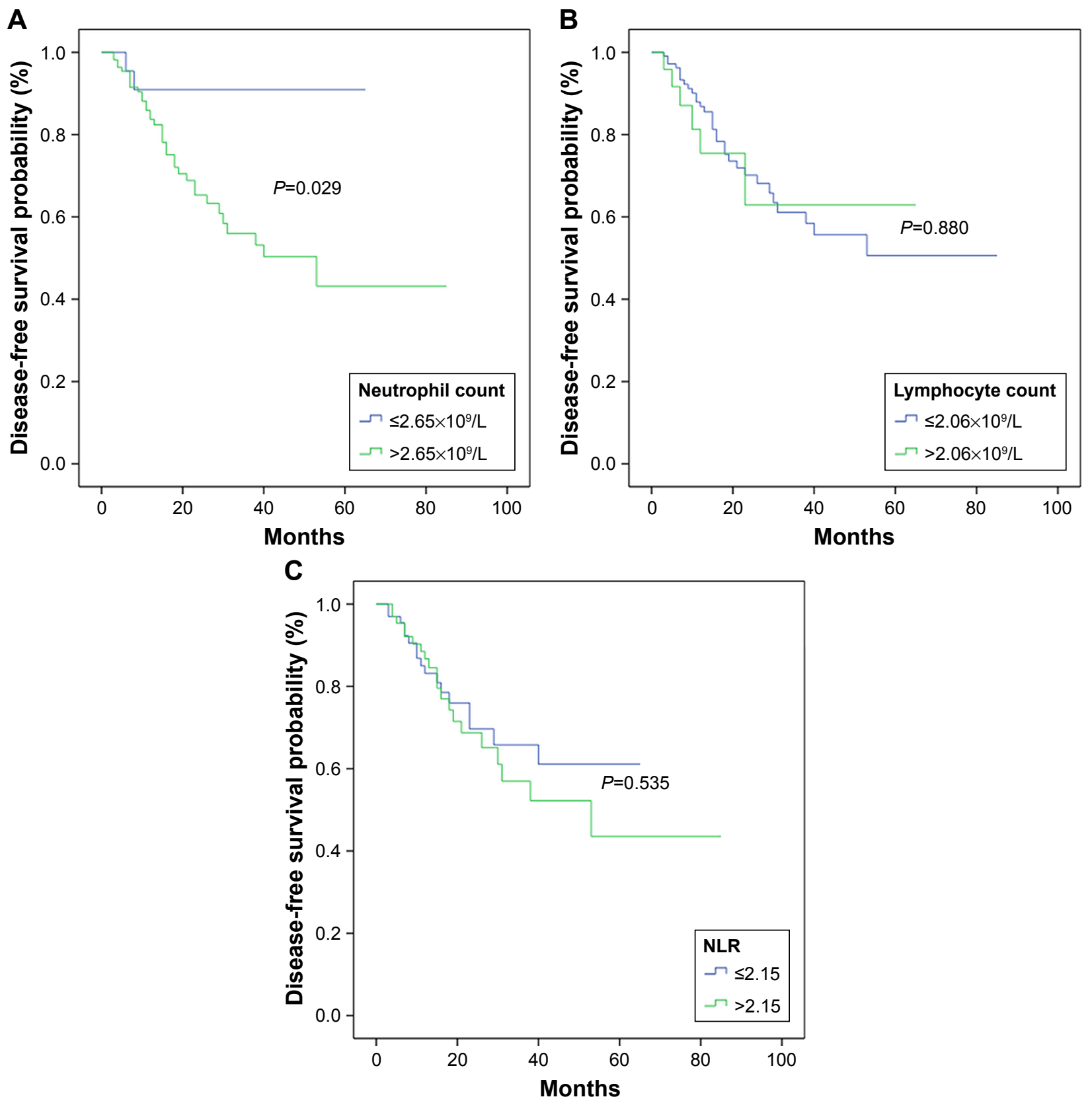

Figure 2 The disease-free survival curves for different inflammatory/immune indicators.

Notes: Among $13 \mathrm{I}$ patients, disease-free survival $(P=0.046)$ was longer in patients with a lower neutrophil count $\left(\leq 2.65 \times 10^{9} / L\right)$ than those with a significantly high neutrophil count (A). However, no significant difference was observed between disease-free survival and lymphocyte count (B), or NLR (C).

Abbreviation: NLR, neutrophil-to-lymphocyte ratio.

tumor metastasis. Neutrophil-derived proteinases, including poly-morphonuclear leukocyte elastase, and matrix metalloproteinases, can remodel the extracellular matrix, which eventually results in the dissociation of tumor cells from the primary tumor to the peripheral blood and distant organs. ${ }^{40}$ The consensus finding in our study was that the high neutrophil count was significantly associated with lymph vessel invasion, which was determined by the presence of neoplastic cell emboli within spaces. We know that the more the invasion of malignant cells on microvessels, the more the likelihood of tumor cells spreading to the peripheral blood and then to distant sites ${ }^{41}$ therefore, the neutrophil count associated with lymphovascular invasion may influence the cancer outcome, mainly due to its correlation with tumor invasion and metastasis, independent of the impact of the treatment. In addition, previous studies have reported that high NLR 
may have a negative impact on clinical outcome, ${ }^{42}$ especially in patients with the luminal A subtype. ${ }^{43}$ The baseline NLR showed no significant association with DFS in the present study. The conflicting findings may be caused by the bias in patient collection.

To the best of our knowledge, this work is the first study that proved the association between a high peripheral lymphocyte count and $\mathrm{pCR}$, and demonstrated the prognostic value of the baseline peripheral neutrophil count in breast cancer patients treated with NAC. Besides, it attributed the relevance of a low NLR to pCR to the high lymphocyte count, which suggested the baseline peripheral lymphocyte count as a more accurate predictive indicator of pCR than NLR. The limitations of our study are listed as follows: First, as a retrospective analysis, records on some parameters were incomplete. Also, several patients were lost to follow up and filtered out from our study. Rigorous prospective studies are therefore needed to extend our findings. Second, the correlation between tumor-infiltrating immune cells and the peripheral immune/ inflammatory biomarkers was not assessed in the present study. The unavailability of the information on circulating tumor cell level before and after NAC also limited our evaluation of the systemic efficiency of chemotherapy. Third, the sample size of our analysis was small and the samples were enrolled from a single oncology center, which limited the general applicability of our findings. Finally, the follow-up time was not long enough to make a significant analysis on overall survival. More studies with longer follow-up and high retention rates are needed to support our findings.

\section{Conclusion}

Our study suggested that a high level of baseline peripheral lymphocyte count was an independent predictor of $\mathrm{pCR}$ for breast cancer patients, and a high level of baseline peripheral neutrophil count had a significant contribution to poor DFS. Both indicators can be obtained from the blood tests easily and reflect the whole-body immunity. In addition, the assessment of the 2 immune/inflammatory indicators at initial diagnosis may provide new ideas on treatment guidance. Future cohort studies are still needed to confirm our results.

\section{Acknowledgments}

This work was supported in part by the National Natural Science Foundation of China $(81572607,81572595$, $81572602,81502299,81502286$, and 81771953) and a project funded by the Priority Academic Program Development of Jiangsu Higher Education Institutions (PAPD).
The authors thank Xiaoming Zha, Lin Chen, and Lijun Ling for their contribution to study design and data acquisition.

\section{Disclosure}

The authors report no conflicts of interest in this work.

\section{References}

1. Mamounas EP, Fisher B. Preoperative (neoadjuvant) chemotherapy in patients with breast cancer. Semin Oncol. 2001;28(4):389-399.

2. Fisher B, Gunduz N, Saffer EA. Influence of the interval between primary tumor removal and chemotherapy on kinetics and growth of metastases. Cancer Res. 1983;43(4):1488-1492.

3. Ragaz J, Baird R, Rebbeck P, Goldie J, Coldman A, Spinelli J. Neoadjuvant (preoperative) chemotherapy for breast cancer. Cancer. 1985; 56(4):719-724.

4. Amiri-Kordestani L, Wedam S, Zhang L, et al. First FDA approval of neoadjuvant therapy for breast cancer: pertuzumab for the treatment of patients with HER2-positive breast cancer. Clin Cancer Res. 2014;20(21):5359-5364.

5. Kaufmann M, Hortobagyi GN, Goldhirsch A, et al. Recommendations from an international expert panel on the use of neoadjuvant (primary) systemic treatment of operable breast cancer: an update. J Clin Oncol. 2006;24(12):1940-1949.

6. Masuda H, Baggerly KA, Wang Y, et al. Differential response to neoadjuvant chemotherapy among 7 triple-negative breast cancer molecular subtypes. Clin Cancer Res. 2013;19(19):5533-5540.

7. Liedtke C, Mazouni C, Hess KR, et al. Response to neoadjuvant therapy and long-term survival in patients with triple-negative breast cancer J Clin Oncol. 2008;26(8):1275-1281.

8. Allred DC, Harvey JM, Berardo M, Clark GM. Prognostic and predictive factors in breast cancer by immunohistochemical analysis. Mod Pathol. 1998;11(2):155-168.

9. van 't Veer LJ, Dai H, van de Vijver MJ, et al. Gene expression profiling predicts clinical outcome of breast cancer. Nature. 2002;415(6871): 530-536.

10. Ahr A, Karn T, Solbach C, et al. Identification of high risk breastcancer patients by gene expression profiling. Lancet. 2002;359(9301): 131-132.

11. Eckert LB, Repasky GA, Ulku AS, et al. Involvement of Ras activation in human breast cancer cell signaling, invasion, and anoikis. Cancer Res. 2004;64(13):4585-4592.

12. Hellemans P, van Dam PA, Weyler J, van Oosterom AT, Buytaert P, Van Marck E. Prognostic value of bcl-2 expression in invasive breast cancer. Br J Cancer. 1995;72(2):354-360.

13. Hollstein M, Sidransky D, Vogelstein B, Harris CC. p53 mutations in human cancers. Science. 1991;253(5015):49-53.

14. Pollard JW. Tumour-educated macrophages promote tumour progression and metastasis. Nat Rev Cancer. 2004;4(1):71-78.

15. Coussens LM, Werb Z. Inflammation and cancer. Nature. 2002; 420(6917):860-867.

16. Denkert C, Loibl S, Noske A, et al. Tumor-associated lymphocytes as an independent predictor of response to neoadjuvant chemotherapy in breast cancer. J Clin Oncol. 2010;28(1):105-113.

17. Loi S, Sirtaine N, Piette F, et al. Prognostic and predictive value of tumor-infiltrating lymphocytes in a phase III randomized adjuvant breast cancer trial in node-positive breast cancer comparing the addition of docetaxel to doxorubicin with doxorubicin-based chemotherapy: BIG 02-98. J Clin Oncol. 2013;31(7):860-867.

18. West NR, Milne K, Truong PT, Macpherson N, Nelson BH, Watson PH. Tumor-infiltrating lymphocytes predict response to anthracycline-based chemotherapy in estrogen receptor-negative breast cancer. Breast Cancer Res. 2011;13(6):R126. 
19. Ray-Coquard I, Cropet C, Van Glabbeke M, et al; European Organization for Research and Treatment of Cancer Soft Tissue and Bone Sarcoma Group. Lymphopenia as a prognostic factor for overall survival in advanced carcinomas, sarcomas, and lymphomas. Cancer Res. 2009;69(13):5383-5391.

20. Luo G, Guo M, Liu Z, et al. Blood neutrophil-lymphocyte ratio predicts survival in patients with advanced pancreatic cancer treated with chemotherapy. Ann Surg Oncol. 2015;22(2):670-676.

21. Ku JH, Kang M, Kim HS, Jeong CW, Kwak C, Kim HH. The prognostic value of pretreatment of systemic inflammatory responses in patients with urothelial carcinoma undergoing radical cystectomy. Br J Cancer. 2015;112(3):461-467.

22. Yamanaka T, Matsumoto S, Teramukai S, Ishiwata R, Nagai Y, Fukushima M. The baseline ratio of neutrophils to lymphocytes is associated with patient prognosis in advanced gastric cancer. Oncology. 2007;73(3-4):215-220.

23. Kishi Y, Kopetz S, Chun YS, Palavecino M, Abdalla EK, Vauthey JN. Blood neutrophil-to-lymphocyte ratio predicts survival in patients with colorectal liver metastases treated with systemic chemotherapy. Ann Surg Oncol. 2009;16(3):614-622.

24. Teramukai S, Kitano T, Kishida Y, et al. Pretreatment neutrophil count as an independent prognostic factor in advanced non-small-cell lung cancer: an analysis of Japan Multinational Trial Organisation LC00-03. Eur J Cancer. 2009;45(11):1950-1958.

25. Seah JA, Leibowitz-Amit R, Atenafu EG, et al. Neutrophil-lymphocyte ratio and pathological response to neoadjuvant chemotherapy in patients with muscle-invasive bladder cancer. Clin Genitourin Cancer. 2015;13(4):e229-e233.

26. Chen Y, Chen K, Xiao X, et al. Pretreatment neutrophil-to-lymphocyte ratio is correlated with response to neoadjuvant chemotherapy as an independent prognostic indicator in breast cancer patients: a retrospective study. BMC Cancer. 2016;16:320.

27. Asano Y, Kashiwagi S, Onoda N, et al. Predictive value of neutrophil/ lymphocyte ratio for efficacy of preoperative chemotherapy in triplenegative breast cancer. Ann Surg Oncol. 2016;23(4):1104-1110.

28. Eryilmaz MK, Mutlu H, Salim DK, Musri FY, Tural D, Coskun HS. The neutrophil to lymphocyte ratio has a high negative predictive value for pathologic complete response in locally advanced breast cancer patients receiving neoadjuvant chemotherapy. Asian Pac J Cancer Prev. 2014;15(18):7737-7740.

29. Suppan C, Bjelic-Radisic V, La Garde M, et al. Neutrophil/lymphocyte ratio has no predictive or prognostic value in breast cancer patients undergoing preoperative systemic therapy. BMC Cancer. 2015; $15: 1027$.
30. von Minckwitz G, Untch M, Blohmer JU, et al. Definition and impact of pathologic complete response on prognosis after neoadjuvant chemotherapy in various intrinsic breast cancer subtypes. J Clin Oncol. 2012;30(15):1796-1804.

31. Nakatsukasa K, Koyama H, Oouchi Y, et al. Docetaxel, cyclophosphamide, and trastuzumab as neoadjuvant chemotherapy for HER2positive primary breast cancer. Breast Cancer. 2017;24(1):92-97.

32. Joseph N, Dovedi SJ, Thompson C, et al. Pre-treatment lymphocytopaenia is an adverse prognostic biomarker in muscle-invasive and advanced bladder cancer. Ann Oncol. 2016;27(2):294-299.

33. Miyashita M, Sasano H, Tamaki K, et al. Tumor-infiltrating CD8+ and FOXP3+ lymphocytes in triple-negative breast cancer: its correlation with pathological complete response to neoadjuvant chemotherapy. Breast Cancer Res Treat. 2014;148(3):525-534.

34. Liu F, Lang R, Zhao J, et al. CD8(+) cytotoxic T cell and FOXP3(+) regulatory $\mathrm{T}$ cell infiltration in relation to breast cancer survival and molecular subtypes. Breast Cancer Res Treat. 2011;130(2):645-655.

35. Coussens LM, Pollard JW. Leukocytes in mammary development and cancer. Cold Spring Harb Perspect Biol. 2011;3(3):a003285.

36. Farrar JD, Katz KH, Windsor J, et al. Cancer dormancy. VII. A regulatory role for CD8+ T cells and IFN-gamma in establishing and maintaining the tumor-dormant state. J Immunol. 1999;162(5):2842-2849.

37. Ladoire S, Arnould L, Apetoh L, et al. Pathologic complete response to neoadjuvant chemotherapy of breast carcinoma is associated with the disappearance of tumor-infiltrating foxp3+ regulatory $\mathrm{T}$ cells. Clin Cancer Res. 2008;14(8):2413-2420.

38. Wislez M, Antoine M, Rabbe N, et al. Neutrophils promote aerogenous spread of lung adenocarcinoma with bronchioloalveolar carcinoma features. Clin Cancer Res. 2007;13(12):3518-3527.

39. Droin N, Hendra JB, Ducoroy P, Solary E. Human defensins as cancer biomarkers and antitumour molecules. J Proteomics. 2009;72(6): 918-927.

40. Gregory AD, Houghton AM. Tumor-associated neutrophils: new targets for cancer therapy. Cancer Res. 2011;71(7):2411-2416.

41. Schoppmann SF, Bayer G, Aumayr K, et al; Austrian Breast and Colorectal Cancer Study Group. Prognostic value of lymphangiogenesis and lymphovascular invasion in invasive breast cancer. Ann Surg. 2004;240(2):306-312.

42. Azab B, Bhatt VR, Phookan J, et al. Usefulness of the neutrophil-tolymphocyte ratio in predicting short- and long-term mortality in breast cancer patients. Ann Surg Oncol. 2012;19(1):217-224.

43. Noh H, Eomm M, Han A. Usefulness of pretreatment neutrophil to lymphocyte ratio in predicting disease-specific survival in breast cancer patients. J Breast Cancer. 2013;16(1):55-59.
OncoTargets and Therapy

\section{Publish your work in this journal}

OncoTargets and Therapy is an international, peer-reviewed, open access journal focusing on the pathological basis of all cancers, potential targets for therapy and treatment protocols employed to improve the management of cancer patients. The journal also focuses on the impact of management programs and new therapeutic agents and protocols on

\section{Dovepress}

patient perspectives such as quality of life, adherence and satisfaction The manuscript management system is completely online and includes a very quick and fair peer-review system, which is all easy to use. Visit http://www.dovepress.com/testimonials.php to read real quotes from published authors. 